\title{
Concepção de uma Família de Gestos para Construção de Objetos Geométricos e sua Utilização em um Sistema de
}

\section{Geometria Interativa para Dispositivos Móveis: GeoTouch}

Conception of a family of gestures for geometric objects construction and its utilization in an interactive geometry system for mobile devices: GeoTouch

\author{
Helena M. Reis \\ ICMC - Universidade de São Paulo \\ helenamcd@gmail.com \\ Seiji Isotani \\ ICMC - Universidade de São Paulo \\ sisotani@icmc.usp.br \\ Leônidas O. Brandão \\ IME - Universidade de São Paulo \\ leo@ime.usp.br
}

\author{
Ramilio R. R. Filho \\ Faculdade de Tecnologia de Taquaritinga \\ ramilio.ramalho@hotmail.com \\ Wilmax M. Cruz \\ ICMC - Universidade de São Paulo \\ wilmaxmcruz@gmail.com \\ Anarosa A. F. Brandão \\ POLI - Universidade de São Paulo \\ anarosa.brandao@poli.usp.br
}

\begin{abstract}
Resumo Os sistemas de Geometria Interativa (SGI) foram desenvolvidos com o objetivo de possibilitar aos estudantes explorarem a geometria por meio do computador, permitindo a construção e manipulação de objetos geométricos, como retas, pontos e circunferências. Tradicionalmente nos SGI, o usuário-aprendiz constrói um objeto geométrico usando o ícone a ele associado disponivel na interface do sistema. Se de um lado a literatura indica que este tipo de sistema pode facilitar o aprendizado de Geometria, de outro existem estudos sugerindo que a redução de icones na interface pode ser benéfica para os usuários iniciantes, diminuindo a dificuldade de se localizar o ícone desejado em meio a vários outros. Esta dificuldade, além de gerar frustração e consequente desmotivação, pode desviar o foco da aprendizagem, com o aprendiz esforçando-se para aprender a usar o sistema e não aos conceitos geométricos. Neste contexto, o presente artigo propõe a redução de icones nas interfaces dos SGI por meio da definição de uma familia de gestos naturais que podem ser utilizados para construção e manipulação de objetos geométricos. Estes gestos foram projetados para, além de reduzir o número de elementos visuais em tela, facilitar também a aprendizagem dos conceitos geométricos. Para validar os gestos propostos desenvolveu-se um SGI utilizando Interface Gestual para uso em dispositivos móveis chamado Geotouch. Foram realizados testes de usabilidade com o Geotouch e sua comparação com outros três SGI para dispositivos móveis disponíveis até o momento. Os resultados encontrados indicam que a interface do GeoTouch, que utiliza a família de gestos propostos, é mais fácil de aprender, possui menor número de problemas de usabilidade e o grau de severidade dos problemas encontrados afetam minimamente o usuário.
\end{abstract}

Palavras-Chave: sistema de geometria interativa, interfaces gestuais, dispositivos móveis, geotouch, interface, usabilidade 
Abstract Interactive Geometry (GI) software were developed with the goal of enabling students to explore geometry through the computer, allowing the construction and manipulation of geometric objects, such as, lines, points and circumferences. Traditionally, while using GI systems, the userlearners build a geometric object using the icon associated with it and available in the system interface. Literature indicates that this type of system can facilitate the learning of geometry. Nevertheless, there are studies suggesting that the reduction of icons in the interface can be beneficial for novice users, reducing the difficulty of locating the desired icon among several ones. This difficulty, beyond generating frustration and consequent lack of motivation, can divert the focus of learning, with the learners struggling to learn how to use the system and not to the geometric concepts. In this context, this paper proposes the reduction of icons at GI systems interfaces through the definition of a family of natural gestures that can be used to build and manipulate geometric objects. These gestures are designed to not only reduce the number of visual elements in screen, but also facilitate the learning of geometric concepts. To validate the proposed gestures we developed a GI system using Gestural Interface for mobile devices called GeoTouch. Usability tests were performed with Geotouch as well as the comparison of it with other three GI systems for mobile devices available to date. Results indicate that the GeoTouch interface, which uses the family of proposed actions, is easier to learn, has fewer usability problems and the severity of the problems encountered affect minimally the user.

Keywords: interactive geometry system, gestural interfaces, mobile devices, geotouch, interface, usability 


\section{Introdução}

Os softwares de Geometria Interativa (GI) são ferramentas que auxiliam no ensino da geometria por meio de computadores. As interfaces desses softwares são importantes, pois são por meio delas que os estudantes conseguem visualizar as informações e interagir com os elementos do sistema.. Com um sistema de GI, o aprendiz pode explorar com o computador construções geométricas do tipo régua-e-compasso, podendo interagir com elas [19, 31, 39]. Essas construções são realizadas a partir de objetos geométricos (e.g. retas, pontos e circunferências), podendo existir vínculos (relações) entre os objetos criados [3, 11, 19, 29, 31]. Na GI esses vínculos são essenciais por permitirem ao sistema redesenhar toda a construção, preservando todas as propriedades da construção inicial [34].

O modelo de interação implementado pela maioria dos sistemas de GI disponíveis até o momento é fortemente baseado no teclado-mouse e interfaces com menu de ícones representando os objetos geométricos ou operações sobre eles [17, 27, 28, 29, 30, 39]. Um exemplo de interface com dezenas de ícones para construção de objetos geométricos, comumente utilizado por sistemas de GI, é apresentado na Figura 1.

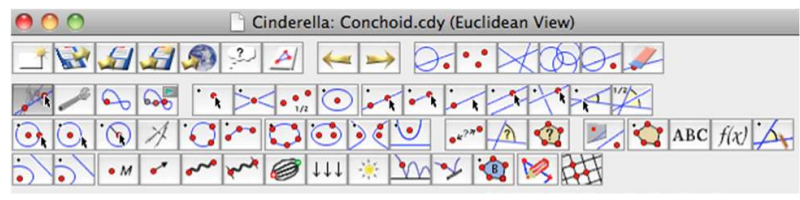

Figura 1. Exemplo de interface tradicional de sistemas de GI baseada em ícones.

Esse poluição de ícones e o grande número de ações/opções apresentados nas interfaces dos sistemas de GI pode dificultar a realização de atividades de cunho pedagógico, principalmente quando pensamos e alunos/usuários iniciantes. Esta dificuldade foi constatada em alguns estudos que analisaram o número de ícones disponíveis na interface de sistemas de GI, como em Schimpf e Spannagel [37] e Reis et al. [18]. A existência de ícones semelhantes e sem significado para o usuário geram erros de operação e, como consequência, induzem a frustração e desinteresse do aluno tanto para com o sistema de GI quanto para o aprendizado de geometria.

Para lidar com esse problema nos anos 2000, apareceu o sistema RISKO [32, 33], que aboliu o emprego de ícones em favor de uma metáfora de instrumentos de desenho geométrico, como o esquadro, o compasso e o lápis. Entretanto o sistema RISKO é destinado primordialmente ao desenho geométrico, não apresentando as propriedades de vínculo entre objetos como na GI. Uma outra proposta para reduzir a dificuldade provocada pelo número de ícones disponíveis é o emprego de gestos [36, 39], principalmente em dispositivos com telas sensíveis ao toque $[35,39]$

Com a evolução e disseminação das tecnologias móveis, torna-se fundamental a necessidade de repensar a interface e a forma de interação com sistemas de GI tornando-a visualmente mais simples [14]. Neste contexto, o foco deste artigo é apresentar o desenvolvimento de um dicionário de gestos para telas de toques e que foram baseados em conceitos geométricos, resultando em um novo sistema de GI, GeoTouch, para dispositivos móveis, cuja forma de interação está baseada em gestos/toques. Deste modo, o aprendiz pode realizar construções geométricas sem a necessidade de encontrar e ativar um ícone relacionado. Para avaliar a nossa proposta foram realizados testes heurísticos de usabilidade para validação da interface do GeoTouch com especialistas em Engenharia de Software.

O restante deste trabalho está organizado da seguinte forma: na Seção 2 é apresentada uma análise dos trabalhos relacionados. Na Seção 3 são identificados os requisitos de um software de GI com interfaces gestuais, que embasaram a definição dos gestos a serem desenvolvidos. A Seção 4 descreve o GeoTouch, um software de GI que usa o catálogo de gestos e a identificação dos requisitos apresentados na Seção 3. Na Seção 5 e 6 é apresentada e analisada uma prova de conceito para avaliar a usabilidade do GeoTouch, comparando-o com outros softwares existentes. Finalmente, na Seção 7, são apresentadas as conclusões do trabalho acompanhadas das considerações finais.

\section{Trabalhos relacionados}

Para identificar de maneira sistemática os trabalhos relacionados com métodos de interação e interfaces para sistemas de GI, realizou-se um mapeamento sistemático cujos objetivos principais foram: (i) analisar quais tipos de interfaces (e formas de interação) vem sendo desenvolvidas para os softwares de GI; e (ii) destacar em quais dispositivos estas interfaces são comumente utilizadas. Esse mapeamento [17], cobriu 998 artigos publicados pela comunidade em mais de 10 anos de pesquisa na área de geometria interativa. Dentre estes estudos 45 deles estavam, de alguma forma, relacionados com interação e interfaces de sistemas de GI. Contudo, apenas 20 deles apresentavam pesquisas na área alvo deste trabalho.

A tabela disponibilizada no endereço http://www.icmc.usp.br/e/2dacf apresenta a lista dos estudos primários selecionados, com os campos: autor, título do artigo, tipo de pesquisa, e tipo de contribuição. Os resultados completos dos trabalhos relacionados identificados por meio do mapeamento sistemático foram 
publicados no Simpósio Brasileiro de Informática na Educação e estão disponíveis em [17].

Em síntese, a Figura 2 mostra a categorização dos trabalhos de acordo com as formas de interação (entrada e saída de dados) com as interfaces dos softwares de GI encontrada nos trabalhos analisados. É importante salientar que um trabalho pode ser classificado em mais de uma categoriza. Observa-se que a maioria dos estudos encontrados preocupa-se em explorar a interação por meio do "Teclado e mouse", sendo que outros tipos de interação, como "Baseada em toque" ou Háptica são ainda pouco exploradas.

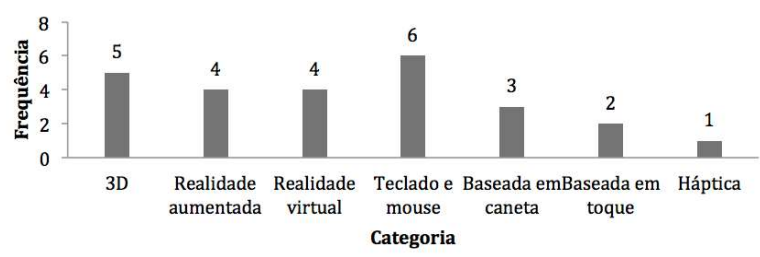

Figura 2. Trabalho relacionados categorizados de acordo com as formas de interação [17]

Além disso, foram identificados também os diferentes tipos de dispositivos nos quais os softwares de GI são executados. A Figura 3 apresenta todos os tipos de dispositivos que foram utilizados nos trabalhos analisados. Observa-se que existem poucos softwares de GI desenvolvidos para outros dispositivos além dos computadores de mesa.

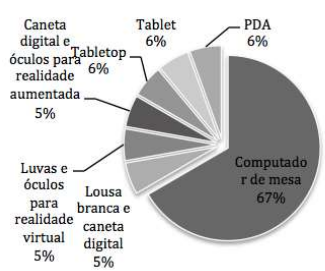

\begin{tabular}{l|c}
\hline Dispositivo & Qtde \\
\hline Computador de mesa & 12 \\
Lousa branca e caneta digital & 1 \\
Luvas e oculos para realidade virtual & 1 \\
Caneta digital e óculos para realidade aumentada & 1 \\
Tabletop & 1 \\
Tablet & 1 \\
PDA & 1 \\
\hline
\end{tabular}

Figura 3. Trabalho relacionados categorizados por tipo de dispositivo utilizado [17]

Por meio desta análise da literatura verificou-se a carência de pesquisas empíricas sobre (i) os benefícios (ou malefícios) oriundos da redução da quantidade de ícones nas interfaces dos sistemas de GI; (ii) modelos de interação para interfaces com tela multitoque; (iii) criação de sistemas de GI para dispositivos móveis. Destaca-se ainda, que os resultados deste mapeamento apresentam indícios de que poucos estudos foram realizados focando no uso de técnicas e metodologias de Interação HumanoComputador para o desenvolvimento de interfaces para softwares de GI.

Dentro os trabalhos analisados, destaca-se o estudo conduzido por de Blagojevic et al. [25] onde são explorados o desenho de objetos geométricos por meio de ferramentas tradicionais como régua, compasso ou transferi- dor na superfície da tela do tablet. Embora o estudo não ser exclusivamente sobre softwares de GI, os autores indicam que esta ferramenta pode também ser utilizada para o ensino da geometria utilizando figuras interativas. Um outro estudo interessante é apresentado por Blanke e Schneider [26] onde foi desenvolvido um software para Tabletop em que os alunos podem interagir com os objetos por meio de uma interface multitoque. Contudo, este trabalho não permite a livre criação de construções geométricas como aquelas disponíveis nos softwares de GI convencionais e nem propõe gestos para manipular estes objetos. Mais recentemente, um estudo conduzido por [23] explorou a relação entre gestos e conceitos geométricos (e.g. circunferências, pontos, retas paralelas). Os autores investigaram o impacto da interação (incluindo gestos) do aluno com os objetos geométricos e a fixação de conhecimentos relacionados a propriedades geométricas. Os resultados obtidos mostraram que a maneira como a interação é definida pode afetar positiva ou negativamente o processo de ensino-aprendizagem. Apesar dos benefícios apresentados, este estudo não focou no desenvolvimento, implementação e avaliação de gestos para construção de objetos geométricos em ambientes de GI.

Com relação as sistemas de GI existentes que contemplam algum tipo de interação multitoque com algum gesto para interação com objetivos geométricos. Um estudo conduzido por Reis et al. [24] identificou três softwares desenvolvidos e disponíveis até o momento: Sketchometry $^{1}$, Geogeobra ${ }^{2}$ e GeometryPad ${ }^{3}$.

Considerando a carência de softwares de GI para dispositivos móveis que adotam o uso de gestos para apoiar construções geométricas, os benefícios oriundos da redução da quantidade de ícones em tela e à crença de que a associação dos gestos a conceitos matemáticos subjacentes às construções geométricas apresenta vantagens ao aprendizado este estudo propõe o desenvolvimento de um software de GI para dispositivos móveis, que utiliza a interação por meio de gestos para a aprendizagem da geometria. Estes gestos foram desenvolvidos de acordo com os conceitos matemáticos subjacentes, sendo o seu desenvolvimento detalhado nas próximas seções.

\section{Requisitos para Definição de Gestos}

A partir dos resultados apresentados em [18; 24], realizou-se entrevistas com especialistas da área de matemática, a fim de investigar quais atividades são realizadas dentro do ambiente de ensino para apoiar a aprendizagem de geometria utilizando um software de GI. Nesta fase, quatro professoras com doutorado em Educação Matemática com mais de 20 anos de atuação profissional foram

\footnotetext{
${ }^{1} \mathrm{http}: / /$ sketchometry.org

${ }^{2}$ http://www.geogebra.org/

${ }^{3}$ http://www.stemonmobile.com/geometry-pad/
} 
entrevistadas individualmente (Apêndice C) durante o período de aproximadamente uma hora. Para conduzir as entrevistas foi definido um conjunto de perguntas-padrão, caracterizando-se assim por uma entrevista estruturada. Além disso, foi dada a liberdade para o especialista expor seus anseios e necessidades sobre o uso da tecnologia, em particular, softwares de GI em dispositivos móveis no contexto escolar.

Os dados das entrevistas foram transcritos e tabulados para auxiliar na definição tanto da família de gestos para construção de objetos geométricos quanto dos requisitos de um sistema de GI para dispositivos móveis. Em particular, as entrevistas apontaram que o aprendizado dos conceitos geométricos pode ser dificultado se os gestos criados não tiverem relação com as propriedades geométricas dos objetos corroborando com as ponderações feitas por [23]. Por exemplo, se uma circunferência for desenhada com o gesto de um círculo, o aluno não vai associar que toda circunferência possui um raio de tamanho $r$ e que todos os pontos desta circunferência estão à distância $r$ do seu centro (ou seja, são equidistantes do centro), gerando problemas conceituais que podem afetar a aprendizagem. Para amenizar este problema, é importante a significância de cada gesto realizado, ou seja, que os conceitos matemáticos subjacentes à construção sejam utilizados para definir um gesto. Além dos benefícios pedagógicos que esta abordagem oferece, também existem vantagens relacionadas ao desenvolvimento de uma interface de GI, pois o estudante pode aplicar o conhecimento prévio sobre geometria para interagir com o software e realizar suas ações.

Além da identificação das funcionalidades mais comumente utilizadas durante as atividades apontadas pelas professoras entrevistadas (i.e. criação e edição de circun- ferências, retas, semirretas, segmento de reta, pontos), também foram levantados requisitos de acordo com as versões disponíveis de softwares de GI. Com estas informações, foi possível elaborar o diagramas de casos de uso e definir as funcionalidades principais para a concepção de novas formas de interagir com softwares de GI que serão apresentados nas seções seguintes.

Com base nas funcionalidades e requisitos identificados, foi elaborado um dicionário de gestos para realizar a construção de objetos geométricos e interagir com o sistema. O dicionário de gestos é composto pela descrição textual do objeto, sua relação com os conceitos geométricos (caso exista) e um conjunto de figuras ilustrativas que apresentam como realizar os gestos em uma interface baseada em tela multitoque. Inicialmente foram gerados múltiplos gestos para atender uma mesma funcionalidade ou requisito e, após múltiplas prototipações e interações com usuários, foram definidas três categorias de gestos para sistematizar a forma de como interagir com o sistema e permitir um alto grau de flexibilidade e extensibilidade.

A descrição e representação sucinta de alguns gestos está disponível na Figura 4. Maiores detalhes sobre a definição do dicionário de gestos está disponível no endereço http://www.icmc.usp.br/e/f92e5. A categoria dos gestos são apresentadas a seguir:

Gestos do Núcleo (ou Gesto Base): Os gestos da categoria "Gestos do Núcleo" são gestos de base do sistema que fornecem suporte para a criação de gestos mais avançados. Nesta categoria, são encontrados os gestos toque breve, pressionar, movimento, arrasto, rotação e ajustar, sendo detalhados a seguir.

Gestos de Navegação: gestos para a navegação no

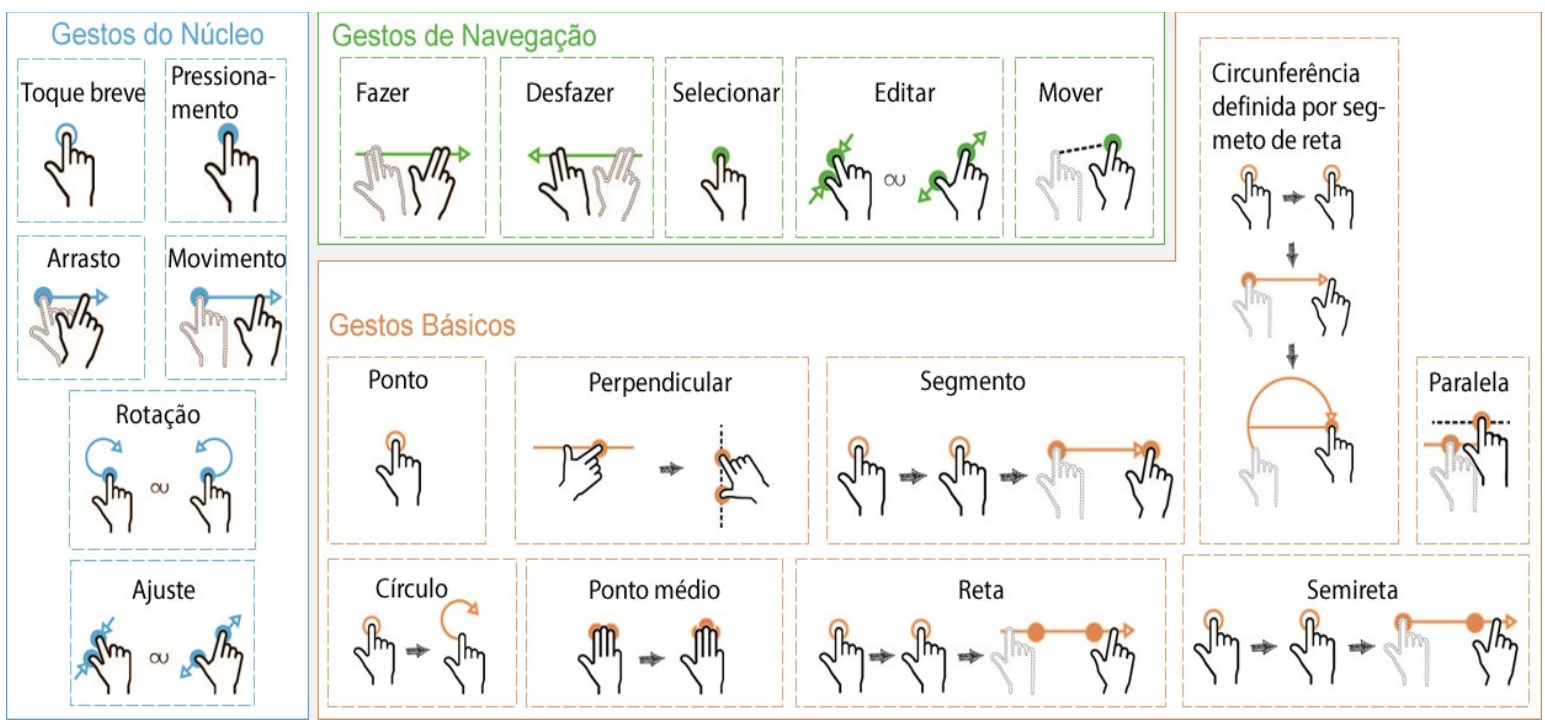

Figura 4. Família de Gestos para Interação com Sistemas de GI 
software, os quais agrupam gestos utilizados para realizar atividades de manipulação/edição e são baseados nos gestos definidos pela categoria "Gestos de Núcleo". Os gestos desta categoria estão relacionados às funcionalidades de desfazer, refazer, selecionar, movimentar e editar.

Gestos Básicos de Construção: Os gestos da categoria "Gestos Básicos" correspondem aos gestos necessários para a criação dos objetos geométricos simples. Assim, nesta categoria são encontrados os gestos de criação para ponto, ponto médio, circunferência, reta, segmento de reta, semirreta, paralela, perpendicular, e etc. Todos os movimentos são baseados em gestos da categoria "Gestos do Núcleo" e foram criados de forma a ter relação direta com os conceitos geométricos envolvidos com a figura a ser construída. Vídeo ilustrativos foram criados utilizando os gestos presentes na Figura 4 para compor o dicionário de gestos de interação:

- Pontos: http://www.icmc.usp.br/e/6c27c

- Reta: http://www.icmc.usp.br/e/808a2

- Seguimento de reta: http://www.icmc.usp.br/e/6df4a

- Circunferência: http://www.icmc.usp.br/e/191ae

\section{GeoTouch: Geometria Interativa para Dispositivos Móveis}

Utilizando o dicionário de gestos desenvolvido neste trabalho, foi proposta a criação de um software de Geometria Interativa para dispositivos móveis com tela multitouch, chamado de GeoTouch [40]. A versão inicial foi desenvolvida na plataforma Android versão 4.0 e pode ser executada em dispositivos móveis, como tablets de 7 e 10 polegadas ou smartphones. Atualmente, o GeoTouch está disponível gratuitamente na loja virtual de aplicativos da Google (o Google Play) e pode ser acessado por meio do link: http://www.icmc.usp.br/e/f4665.
O Geotouch possui todas as funcionalidades que envolvem construções básicas de objetos geométricos para o ensino da geometria euclidiana. Além da construção destes objetos, também é possível a sua manipulação (e.g. movimentação de um objeto) e edição (e.g. aumentar o tamanho de uma reta ou circunferência). Outras funcionalidades elementares também foram desenvolvidas (e.g. Novo, Abrir, Salvar, Exibir Eixo Cartesiano, Medir Distância). Um vídeo ilustrativo do uso do GeoTouch está disponível em: http://www.icmc.usp.br/e/185e3

$\mathrm{O}$ software foi organizado em diferentes pacotes. Pacotes são diretórios (ou pastas) que são utilizados para agrupar classes semelhantes ou relacionadas. Foi desenvolvido dois principais pacotes no software (Figura 5). O principal pacote, chamado de "Pacote Básico", contém as classes para a criação dos objetos geométricos, como, ponto, ponto médio, circunferência, circunferência definida por segmento de reta, reta, semi-reta, segmento de reta, perpendicular, paralela e intersecção. Este mesmo pacote possui um espaço chamado Cache para armazenar todos os objetos geométricos construídos até o momento. Este espaço é importante, pois caso o estudante mude a orientação do dispositivo móvel (e.g. tela em paisagem ou em retrato), o software seja capaz de apresentar novamente os objetos nas posições corretas. Além disso, a cada momento que se cria um novo objeto, por padrão do Android, a tela é limpada. Para evitar que os objetos construídos sejam removidos, é utilizado o espaço de Cache para apresentar os objetos construídos em tela. $\mathrm{O}$ "Pacote de Ajuda", contém a classe que apresentam as figuras e as descrições dos gestos para utilizar as funcionalidades do software. E, por fim, o "Pacote de Visualização", são as classes que possibilitam a visualização dos elementos em tela e que recebem a resposta de alguma ação realizada na tela do dispositivo móvel.
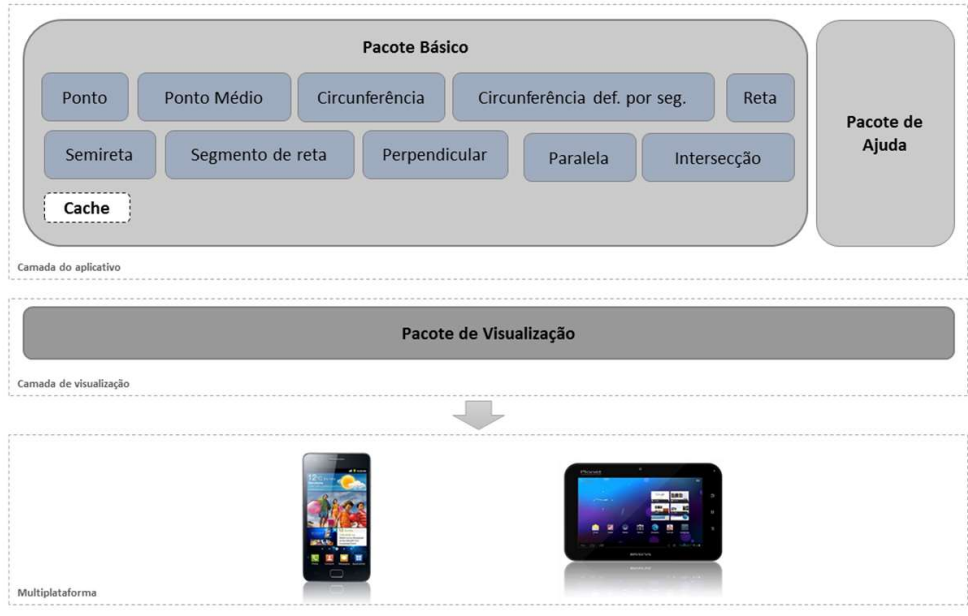

Figura 5. Arquitetura do software GeoTouch. 
A Figura 6 apresenta as classes que estão localizadas no pacote básico da Figura 5. Neste pacote estão localizada as classes dos objetos geométricos, como, ponto, ponto médio, intersecção, reta, semi-reta, segmento de reta, paralela, perpendicular, circunferência e circunferência definida por segmento de reta. Todas estas classes são referenciadas dentro da classe Reconhecimento de Gestos, que é responsável em identificar qual gesto o usuário está realizando (e.g. ponto ou reta) e direcionar para a ação correta (e.g. criar ou mover ou remover). Além disso, as classes para a construção dos objetos geométricos são filhas da classe Objeto Dinâmico, que compartilha características em comum entre as classes, como id, rótulo, ponto, x e y. A classe Circunferência tem como filha a classe Circunferência def. Seg. e possui um atributo para informar o seu raio. A classe Reta tem como filha as classes Semi-reta, Paralela, Segmento de reta e Perpendicular. E, por fim, a classe Ponto tem como filha as classes Intersecção e Ponto Médio, tendo também um atributo para informar o seu raio. Esta classe Ponto é a base de todas as construções dos outros objetos geométri$\cos$.

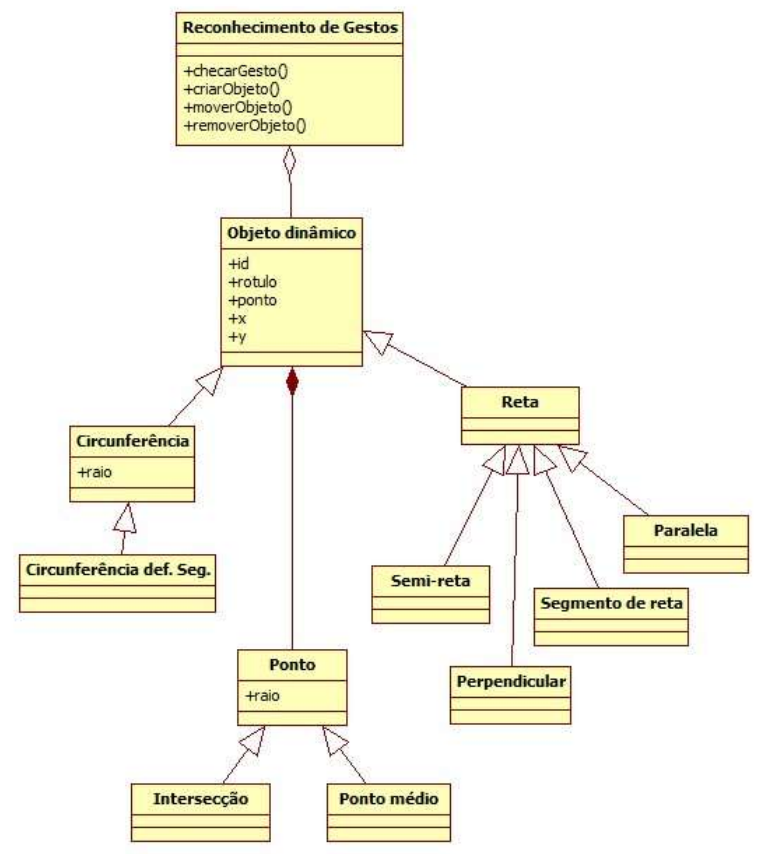

Figura 6. Algumas das classes que presentes no pacote básico para descrever os objetos geométricos.

Os objetos geométricos do software são construídos e exibidos em uma classe que refere-se a área de desenho. Esta classe herda as características de sua classe pai RelativeLayout, que possibilita que um grupo de objetos sejam apresentados em sua posição relativa, ou seja, permite que o objeto seja apresentado em qualquer parte da tela. Por exemplo, o usuário consegue criar um ponto em qualquer lugar que desejar, sem a necessidade de estar alinhado a quaisquer outros objetos.

Os objetos são criados a partir de toques na tela, sendo que um conjunto destes toques compõe os gestos para a execução das funcionalidades. Todo toque em tela é verificado pela classe Reconhecimento de Gestos, que é responsável por identificar o tipo de toque (e.g. toque breve, movimento ou pressionar) e decidir qual gesto será executado (e.g. seleção, movimentação ou criação).

O código a seguir mostra resumidamente a implementação do método que é invocado sempre que ocorrer algum toque em tela. $\mathrm{O}$ toque é verificado em três tipos de ações: ACTION DOWN (pressionamento), ACTION_MOVE (movimentação) e ACTION_UP (despressionamento). $\mathrm{Na}$ ação de pressionamento em tela pelo o usuário, é verificado se algum objeto já foi criado e se esta sendo selecionado; caso isto não ocorra, é armazenado em uma váriavel indicando que o usuário deseja criar um ponto. Caso o usuário esteja movimentando o(s) dedos(s), é verificado se há algum objeto que esteja selecionado para haver a movimentação do mesmo; caso contrario, é verificado se o usuário está realizando o gesto para a criação de circunferência, reta, segmento de reta, semireta, paralela, ponto médio ou perpendicular. E por fim, quando o usuário deixa de tocar a tela (despressionamento), é verificado se o usuário deseja criar algum objeto geométrico e é chamado o método para a criação de objetos geométricos. Um fluxograma deste código está presente Apêndice B.

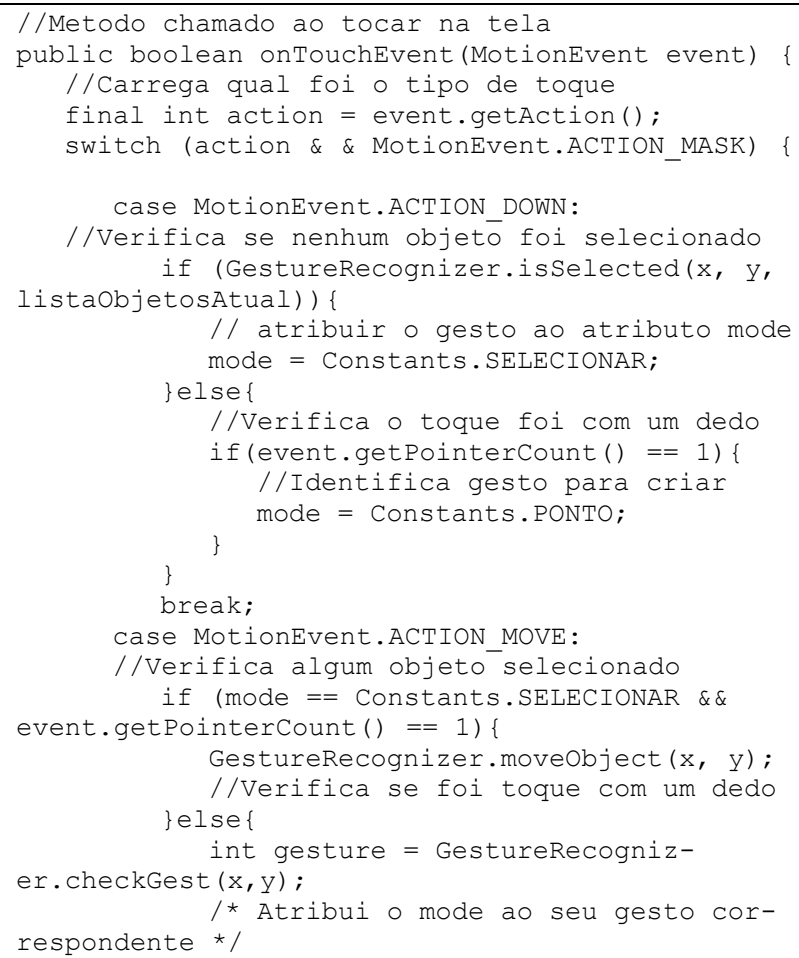




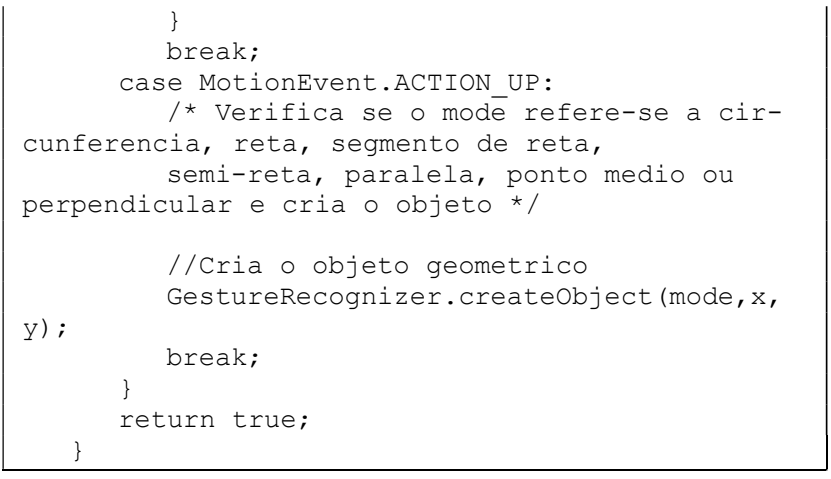

A Figura 7 mostra a tela inicial do GeoTouch com e sem o menu lateral. Este menu (Figura 7(a)) é acessado por meio de um ícone localizado na parte superior esquerda e foi desenvolvido de modo que o usuário consiga escondê-lo quando desejar trabalhar na área de desenho (Figura 7(b)) e mostrá-lo quando precisar acessar alguma de suas funcionalidades. É importante observar que o menu e ícones criados são utilizados apenas para tarefas não relacionadas a construção e edição de objetos geométricos. Isso possibilitou uma sensível redução no número de ícones que deveriam, de outra forma, ser exibidos, além de propor uma forma singular de interagir com softwares de GI.

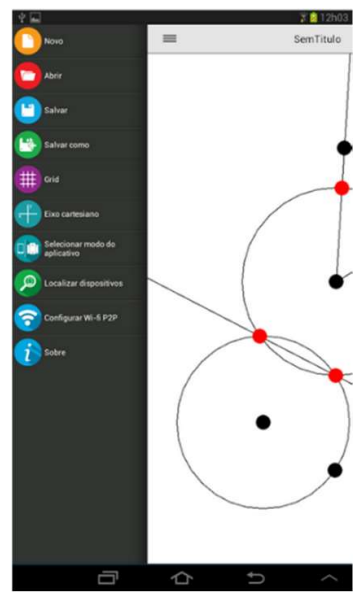

(a)

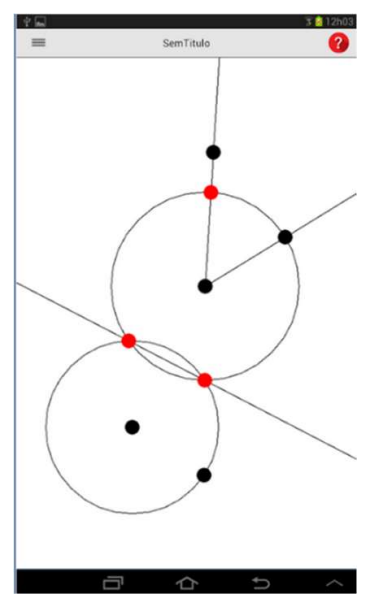

(b)
Figura 7: Tela inicial do software Geotouch.

\section{Prova de conceitos}

Foi realizada uma prova de conceitos com o objetivo de comparar a forma de interação proposta para construção de objetivos geométricos por meio de gestos com o formato padrão de interação utilizado por outros softwares de GI. Além disso, um outro objetivo foi também verificar problemas de usabilidade com o software de GI desenvolvido GeoTouch. Dessa forma, o Geotouch foi analisado conjuntamente com mais três softwares de GI disponíveis para dispositivos móveis apresentados na Seção 2, totalizando quatro softwares selecionados avaliação e comparação: (a) Geotouch, (b) GeometryPad, (c) GeoGebra e (d) Sketchometry.

A técnica selecionada para esta prova de conceitos foi a análise de usabilidade utilizando a avaliação heurística definida por Nielsen [15]. Nielsen define o critério de usabilidade como um conjunto de fatores que qualificam quão bem uma pessoa pode interagir com um sistema computacional. Tais critérios ${ }^{1}$ possuem o objetivo de encontrar problemas de usabilidade, o que ajuda na avaliação da interação da interface do sistema com o usuário e na construção de soluções para o desenvolvimento de melhores interfaces e interações homem-máquina [1, 15].

Segundo Dringus [6] a avaliação heurística pode ser aplicada em interfaces de softwares educacionais, possibilitando a identificação de vários problemas que podem afetar o aprendizado. A avaliação heurística é frequentemente utilizada por pesquisadores que procuram avaliar a usabilidade de uma interface em um curto período de tempo e um custo baixo. Normalmente é conduzido com a participação de 3 a 5 avaliadores, pois segundo diversos pesquisadores, esse número de avaliadores é suficiente para identificar por volta de $80 \%$ de todos os problemas de usabilidade, além de permitir que o processo seja bem documentado e com alto grau de replicabilidade $[1,38]$.

Para conduzir a avaliação heurística foram convidados cinco pesquisadores com pelos menos dois anos de experiência nas áreas de engenharia de software, usabilidade e técnicas de avaliação heurística. Cada um destes pesquisadores avaliou cada software por pelo menos duas horas, totalizando mais quarenta horas de avaliação. Toda a atividade de avaliação foi acompanhada por pelo menos um dos autores deste trabalho e anotações referentes as dificuldades de interação foram realizadas. Para realizar os testes dos softwares utilizou-se como hardware um tablet de 7 polegadas executando o sistema operacional Android. Também foi utilizado um computador, para que os avaliadores pudessem responder um questionário para apontar os problemas de usabilidade e, um guia impresso contendo uma lista de atividades a serem exploradas nos ambientes. $\mathrm{O}$ teste foi realizado em quatro fases como mostra a Figura 8.

\footnotetext{
${ }^{1}$ As dez heurísticas propostas por Nielsen [15] são: (H01) Visibilidade do status do sistema; (H02) Correspondência entre o sistema e o mundo real; (H03) Controle do usuário e liberdade; (H04) Consistência e padrões; (H05) Prevenção de erros; (H06) Reconhecimento em vez de lembrança; (H07) Flexibilidade e eficiência de uso; (H08) Estética e design minimalista; (H09) Ajuda para usuário identificar, diagnosticar e corrigir erros; (H10) Ajuda e documentação
} 


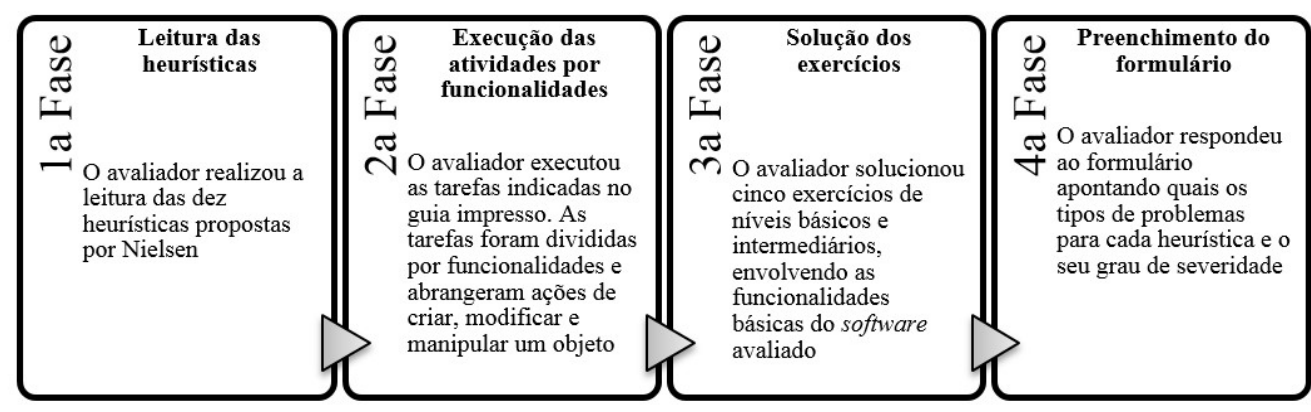

Figura 8. Fases da avaliação heurística no softwares propostos pelos avaliadores.

A primeira fase consistiu na leitura e compreensão das dez heurísticas pelos avaliadores. Como todos os avaliadores tinham conhecimento prévio sobre técnicas de usabilidade, não houve necessidade de treinamento prévio para aplicação das heurísticas de Nielsen.

Em Seguida, os avaliadores realizaram a segunda fase, que consistiu em executar um conjunto de 38 atividades pré-definidas e disponibilizadas como um guia estruturante. Neste guia, cada avaliador teve como tarefa aplicar as dez heurísticas de usabilidade para cada item da lista de atividades. Para cada funcionalidade, foi solicitado que realizassem atividades que envolvessem construção de objetos básicos de geometria. Além disso, foram propostas tarefas padrão, como fazer e desfazer, limpar tela inteira, abrir um documento e salvar um documento. Estas atividades foram agrupadas por tipo de funcionalidade que o software disponibiliza como criação de objetos, edição de objetos e, por fim, tarefas de interação com o sistema. Alguns exemplos destas atividades são apresentados a seguir:

\section{Criar um ponto \\ 2. Mover o ponto \\ 3. Modificar o tamanho do ponto \\ 4. Criar ponto médio forma livre \\ 5. Criar ponto médio por dois pontos \\ 6. Mover ponto médio \\ 34. Limpar tela inteira \\ 35. Excluir mais de um objeto ao mesmo tempo \\ 36. Modificar nome do documento \\ 37. Salvar documento \\ 38. Abrir documento}

$\mathrm{Na}$ terceira fase foi solicitado que os avaliadores resolvessem uma lista de exercícios relacionados a construção geométrica de nível básico e intermediário. O objetivo desta etapa foi simular o uso do software em atividades de aprendizagem de geometria. Para exemplificar uma das atividades solicitadas foi a construção de uma mediatriz para dois pontos criados A e B.

Por fim, na quarta fase o avaliador preencheu o formulário sobre as heurísticas de usabilidade, de acordo com o grau de severidade do problema conforme proposto por [15].

Após os softwares serem avaliados, foi criada uma lista para cada software contendo todos os problemas encontrados e classificados nas heurísticas. É importante ressaltar que devido à complexidade de algumas atividades, os erros e problemas podem ter sido classificados em mais de uma heurística, afetando assim a frequência dos erros/problemas reportados.

\section{Resultados e Discussão}

Inicialmente, para oferecer uma visão geral dos resultados obtidos, apresenta-se a Figura 23 que destaca a frequência de problemas encontrados em cada uma das heurística avaliadas e para cada um dos softwares avaliados. No eixo x, é mostrado a abreviação das heurísticas. No eixo y, é mostrado a quantidade de vezes que o problema foi encontrado.

Na Figura 23, é possível observar que o GeoTouch foi o sistema com menor número de erros/problemas de usabilidade segundo os especialistas. E o GeoGebra foi o sistema que apresentou o maior número de erros/problemas. É possível verificar também que nenhum dos quatro softwares apresentaram problemas relacionados a consistência e padrões (H04). Isso mostra uma certa maturidade dos softwares analisados uma vez que todos eles são versões adaptadas de softwares de geometria interativa desenvolvidos para computadores de mesa. Entretanto, em todos os softwares avaliados, foram encontrados números consideráveis de problemas nas heurísticas H05: problemas relacionados à prevenção de erros; H06: reconhecimento em vez de lembrança; e H07: flexibilidade e eficiência de uso.

$\mathrm{O}$ problema relacionado à prevenção de erros $(\mathrm{H} 05)$ abrange a apresentação de ícones fora do padrão (i.e. ícones comumente usados), falta de descrição das funções e mensagens de erro ou de passo a passo para executar as atividades. Ou seja, o design da interface e a forma de expor seus elementos não evitaram que os avaliadores cometessem erros ao utilizar os softwares. 


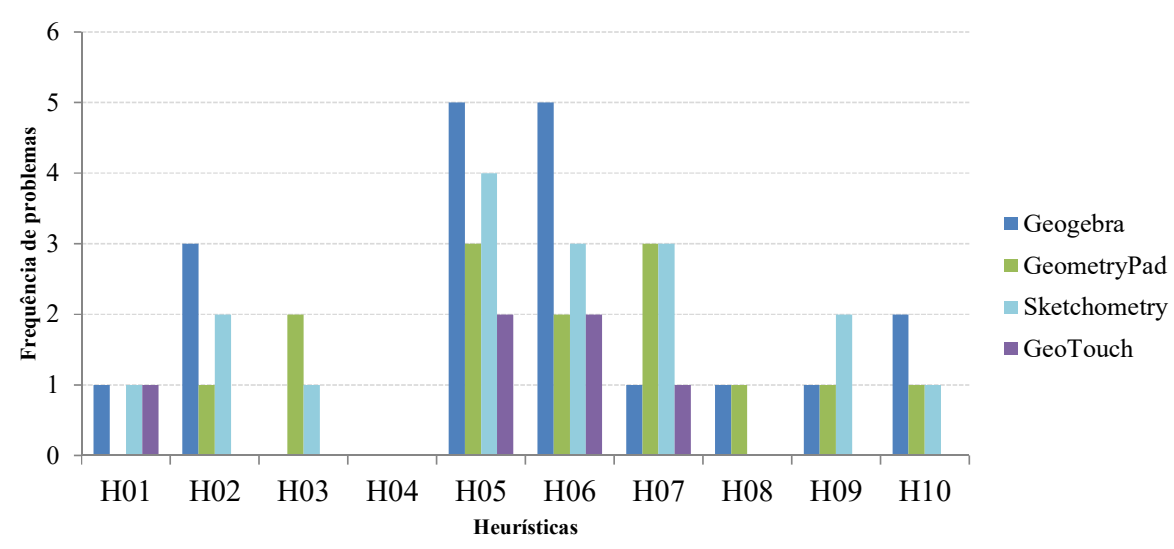

Figura 23: Frequência de problemas encontrados por heurística e por software avaliado.

Geogebra e Geometry Pad não apresentaram nenhuma tecla de atalho, afetando a flexibilidade e eficiência no uso do sistema. Contudo, o único software que apresentou problemas relacionados a estética e design minimalista (H08) foi o Geometry Pad, pois apresentou informação irrelevante na interface, como a funcionalidade de exportação localizada na barra de título. Além disso, o Geometry Pad apresentou um maior número de problemas relacionados ao controle e liberdade do usuário (H03), pois não possibilitou que o usuário excluísse vários objetos ao mesmo tempo.

O software Geogebra apresentou uma maior ocorrência de problemas nas heurísticas H02, H06 e H10, se comparado com os outros softwares. Estes problemas estão relacionados a (i) palavras ou elementos em tela que não são familiares aos usuários; (ii) a dificuldade de lembrar como utilizou uma funcionalidade pela última vez; e (iii) a carência de documentação do software.

Em relação ao Geotouch, um dos problemas identificados foi a falta de informação sobre quais são os objetos que estão sendo criados no momento da sua construção (H01), por meio de uma mensagem ou informação similar. Por exemplo, o gesto para construir uma reta pode ser similar ao gesto para a construção dos gestos semirreta e segmento de reta. Para os avaliadores, é interessante que haja uma mensagem na tela identificando qual objeto será construído com o gesto que está sendo executado no momento.

Outro problema encontrado no Geotouch foi a dificuldade de lembrar os gestos de Desfazer e Refazer (H06), levando um dos avaliadores a consultar a documentação diversas vezes. Por fim, o último problema identificado pelos os avaliadores foi a carência de atalhos para algumas funções do menu lateral, como novo, salvar, salvar como, grid e eixo cartesiano (H07).
Para medir o quanto estes problemas podem afetar a usabilidade de cada software também foi analisado o grau de severidade de cada problema [15]. A Figura 24 apresenta os gráficos que destacam os graus de severidade atribuídos aos problemas identificados pelos especialistas em cada heurística e para cada software. Estes gráficos são apresentados como polígonos de 10 lados em forma de teia de aranha onde cada vértice representa um dos critérios de usabilidade. O grau de severidade para cada critério é indicado fazendo o preenchimento do polígono (de dentro para fora). Segundo Nielsen o grau de severidade pode ser dividido em quatro categorias [15]: 0 - Não concordo que seja um problema de usabilidade $(0 \%$ de severidade); 1 - Cosmético: problema superficial, que precisa ser somente quando houver tempo e dinheiro (25\% de severidade); 2 - Leve: problema de usabilidade pequeno, que possui baixa prioridade para ser solucionado (50\% de severidade); 3 - Sério: problema de usabilidade severo, que possui prioridade e deve ser corrigido assim que possível (75\% de severidade) e; 4 - Crítico: possui prioridade máxima e precisa de intervenção imediata por impedir que os usuários utilizem apropriadamente a interface $(100 \%$ de severidade). Assim, caso o grau de severidade do critério H01 seja leve, preenche-se $25 \%$ deste vértice. 


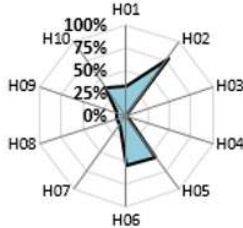

- Geogebra

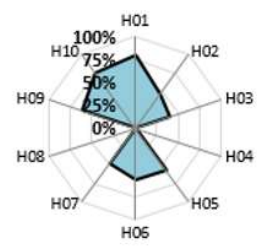

- Sketchometry

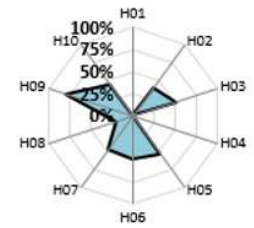

- Geometry Pad

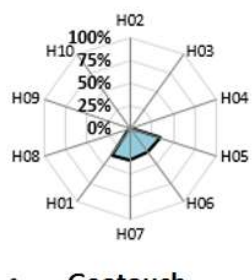

- Geotouch

Figura 24: Grau de severidade entre as heurísticas.

Sobre o Geogebra, é possível notar que a H02 (correspondência entre o sistema e o mundo real) tem maior grau de severidade (corresponde aproximadamente $75 \%$ de severidade na heurística H02), se comparado com os demais problemas. Isto indica que este problema é sério e precisa de atenção imediata. Os problemas classificados nas heurísticas H05 (prevenção de erros) e H06 (reconhecimento em vez de lembrança) estão relacionadas com o segundo maior grau de severidade (aproximadamente $50 \%$ ) e precisam ser analisados em futuras versões do software. Não foram encontrados problemas relacionados com as heurísticas H03 (controle do usuário e liberdade) e H04 (consistência e padrões).

No Geometry Pad, observa-se que a heurística relacionada com a ajuda para identificar, diagnosticar e corrigir erros (H09) possui problemas com maior severidade, apresentando aproximadamente $75 \%$ de grau de severidade da H09 e, portanto, a sua solução é considerada emergencial. As heurísticas relacionadas a controle do usuário e liberdade (H03), prevenção de erros (H05), reconhecimento em vez de lembrança $(\mathrm{H} 06)$, flexibilidade e eficiência de uso (H07) e (H10) ajuda e documentação apresentaram aproximadamente $50 \%$ de severidade e necessitam de atenção. A heurística $\mathrm{H} 08$ apresentou problemas com o menor grau de severidade no sistema, correspondendo aproximadamente a $25 \%$ de severidade, sendo considerada de baixa prioridade. É importante salientar que apesar da frequência de erros neste software ser menor que o do GeoGebra (Figura 23), o grau de severidade destes erros são maiores (Figura 24) indicando que os avaliadores tiveram mais dificuldade de interação ao realizar as tarefas propostas.

No Sketchometry, a heurística relacionadas a visibilidade do status do sistema (H01) e ajuda e documentação (H10) apresentaram alto grau de severidade, correspondendo aproximadamente $75 \%$. Em seguida, as heurísticas

relacionadas a correspondência entre o sistema e o mundo real (H02), prevenção de erros (H05), reconhecimento em vez de lembrança (H06), flexibilidade e eficiência de uso (H07) e ajuda para identificar, diagnosticar e corrigir erros (H09) apresentaram grau de severidade aproximadamente $50 \%$. Apesar deste software apresentar uma menor frequência de erros se comparado ao Geogeobra e o Geometry Pad, a maioria deles possui alto grau de severidade e que pode afetar seu uso e adoção por usuários iniciantes.

Por fim, no Geotouch foram encontrados somente problemas relacionados a visibilidade do status do sistema (H01), prevenção de erros (H05), reconhecimento em vez de lembrança (H06) e flexibilidade e eficiência de uso (H07). Todos os problemas foram classificados como cosmético ou de severidade leve (entre $25 \%$ a $50 \%$ de severidade). Estes resultados indicam que o Geotouch possui a menor frequência de erros e o menor grau de severidade destes erros dentre os quatro softwares avaliados.

Além do teste de usabilidade, ao final de todo o processo, coletou-se a impressão dos avaliadores quanto as atividades realizadas em cada software testado. As perguntas feitas para os avaliadores foram: (Q1) O tamanho da tela é adequado para realizar as atividades?; (Q2) A interação por meio dos dedos foi fácil/intuitivo?; (Q3) A interação para construção dos objetos geométricos são intuitivos/naturais?; (Q4) Os conhecimentos matemáticos previamente conhecidos afetou no modo de construir os objetos geométricos?; (Q5) As telas lhe parecem visualmente agradáveis?; (Q6) A interface está adequada para um dispositivo móvel (ou é semelhante a interface de um software de desktop)?; (Q7) A interface é fácil de aprender?; (Q8) A execução das atividades é rápida?

A Figura 11 apresenta a comparação dos resultados do questionário do software GeoTouch com os softwares GeoGebra, Geometry Pad e Sketchometry. É possível observar que o software GeoTouch apresentou resultados superiores aos outros softwares, exceto a Q7, representando maior satisfação dos avaliadores em relação ao uso do software.

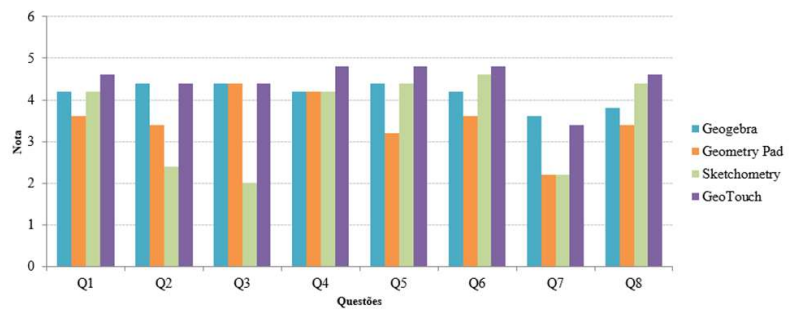

Figura 11: Pontuação final do questionário entre os softwares avaliados. 


\section{Conclusões}

O modelo de interação implementado pela maioria dos sistemas de GI tem sido baseado em menus e ícones representando os objetos ou operações sobre eles. Além deste modelo não ser adequado para dispositivos móveis (que possuem telas do tipo multitoque e pequenas se comparadas aos desktops), diversos estudos apontam que interfaces de GI com muitos ícones podem gerar dúvidas, causar erros de interação e, por consequência, frustrar o aluno (principalmente o iniciante) durante o processo de aprendizagem [18][37].

Devido a estes problemas, o presente trabalho propôs a criação de um dicionário de gestos como meio de interação com o sistema de GI tanto para construção de objetos geométricos quanto para a realização de ações de edição e manipulação sobre os objetos construídos em tela. Este dicionário de gestos está disponível no Apêndice A. Para a definição e desenvolvimento destes gestos, em primeiro lugar, foram realizadas entrevistas com especialistas em ensino de geometria para identificar como são utilizados os conceitos geométricos básicos durante as construções geométricas realizadas em situações de ensino e aprendizagem. Em seguida, gestos que fazem a associação direta com os conceitos geométricos foram definidos para serem utilizados, principalmente, em dispositivos móveis com telas sensíveis ao toque, como tablets e smartphones. Acredita-se que este é a primeira iniciativa de definir e padronizar gestos de interação com ambientes de GI em telas multitoque. Essa crença é fortalecida pelos resultados reportados em uma análise sistemática profunda da literatura que demonstrou que são poucos os trabalhos relacionados a GI em dispositivos móveis, além de indicar uma lacuna de pesquisa na definição de interfaces gestuais e padrões de interação para sistemas de GI [13].

A partir da definição do dicionário de gestos foi desenvolvido o GeoTouch, um software de GI que implementa todas as funcionalidades básicas para construção de objetos geométricos. O GeoTouch está disponível gratuitamente na loja virtual de aplicativos da Google por meio do link: https://goo.gl/Sa7Kdi e um vídeo ilustrando algumas funcionalidades básicas do sistemas está disponível em: http://www.icmc.usp.br/e/185e3

Para avaliar a interface do GeoTouch desenvolvida utilizando os gestos propostos, foi realizada uma prova de conceitos para testar sua usabilidade em conjunto com a interface de três outros softwares de GI: Geogebra, Geometry Pad e Sketchometry (apresentados na Seção 2 deste trabalho). Para esta avaliação adotou-se as heurísticas proposta por Nielsen [15]. Assim, foi aplicado um con- junto de atividades para avaliar, encontrar e analisar a frequência de erros de interação e a severidade destes erros (Seção 5 e 6). Com relação a frequência de erros encontrados, a interface do Geogebra foi o que apresentou maior índice de problemas, 19 erros reportados; sendo que o Skechometry apresentou 17 erros; o Geometry Pad, 14 erros; e o GeoTouch, 6 erros. Enquanto que, em relação ao grau de severidade dos erros, o Geometry Pad foi o que apresentou os piores resultados, onde cinco das dez heurísticas avaliadas tiveram grau de severidade em torno de 50\% (leve), e uma heurística apresentar grau de severidade em torno de $75 \%$ (séria). A interface que apresentou problemas com graus de severidade mais baixos foi o Geotouch. Estes resultados sugerem que a interface proposta, baseada no dicionário de gestos, é mais adequada se comparada às interfaces dos softwares de GI para dispositivos móveis já existentes no mercado.

Acredita-se que o GeoTouch possa se tornar uma ferramenta de referência que pode ser utilizada para auxiliar alunos e professores no processo de ensino e aprendizagem da geometria utilizando dispositivos móveis. Este auxílio se dá não apenas pelos recursos do software, mas também pelo fato de todo o desenvolvimento estar fortemente relacionando aos conceitos geométricos subjacentes à construção dos objetos geométricos, a fim de facilitar o aprendizado da interface e do conteúdo.

Contudo, vale ressaltar que embora a realização da avaliação heurística permitir que os gestos e a interface propostas fossem avaliados no aspecto de usabilidade, observa-se ainda há a necessidade de uma avaliação com alunos e professores em ambientes reais de ensinoaprendizagem, com atividades que os estudantes executam diariamente em sala de aula. Assim, como trabalhos futuros pretende-se realizar experimentos em ambientes reais, com alunos e professores, para validar o ganho pedagógico ao utilizar o Geotouch. Além disso, em futuras versões deste sistema serão desenvolvidas as funcionalidades de criação de ponto sobre objetos, cálculo de ângulos, inclusão de rótulos, compartilhamento e colaboração síncrona de construções geométricas e avalição automática de exercícios.

\section{Agradecimentos}

Os autores deste trabalho agradecem o apoio financeiro condido pelo CNPq, CAPES e FAPESP para a realização desta pesquisa. Estendemos nossos agradecemos aos especialistas que avaliaram os softwares de GI e as professoras entrevistadas durante a concepção dos gestos. Ambos ofereceram contribuições valiosas sem as quais está pesquisa não teria sido concluída com sucesso. 


\section{Referências}

[1] Baker, K.; Greenberg, S.; Gutwin, C. (2001). Heuristic Evaluation of Groupware Based on the Mechanics of Collaboration. In: Proceedings of the IFIP International Conference on Engineering for Human-Computer Interaction, 123-140.

[2] Bowman, C. L. (1985). Individuals and points. Notre Dame J. Formal Logic, 26(1), 61-75.

[3] Erez, M. M.; Yerushalmy, M. (2007). "If You Can Turn a Rectangle into a Square, You Can Turn a Square into a Rectangle ..." Young Students Experience the Dragging Tool. International Journal of Computers for Mathematical Learning, 11(3), 271-299.

[4] Carvalho, B. (1988). Desenho geométrico. 332 páginas.

[5] Coll, C.; Teberosky, A. (2002). Aprendendo Matemática - Conteúdos Essenciais para o Ensino Fundamental. Ática edição. 256 páginas.

[6] Dringus, L. P. (1995). An iterative usability evaluation procedure for interactive online courses. Journal of Interactive Instruction Development, 7(4), 10-14.

[7] Dolce, O.; Pompeu, J. N. (2005). Fundamentos de Matemática Elementar - Geometria Plana. 7 edição. 456 páginas.

[8] Dolce, O.; Iezzi, G.; Machado, A. (2009). Geometria Plana: Conceitos Básicos - Volume único. 208 páginas

[9] Iemenes, L. M. P.; Jakubovic, J.; Lellis, M. C. (1997). Para que serve a Matemática? - Geometria (2o Grau). 48 páginas.

[10] Iezzi, G.; Dolce, O.; Degenszajn, D. M.; Perigo, R. (1997). Matemática: Volume Único. 651 Páginas.

[11] Brandão, L. O. ; Isotani, S. ; Moura, J. G. (2006) Imergindo a Geometria Dinâmica em Sistemas de Educação a Distância: iGeom e SAW. Revista Brasileira de Informática na Educação, 14(1), 41-49.

[12] Kortenkamp, U.; Materlik, D. (2004). Geometry teaching in wireless classroom environments using Java and J2ME. Science of Computer Programming, 53(1), 71-85.

[13] Laborde, C. (2007). The role and uses of technologies in mathematics classrooms: Between challenge and modus vivendi. Canadian Journal of Science, Mathematics and Technology Education, 7(1), 68-92.

[14] Nacenta, M. A.; Kamber, Y.; Qiang, Y.; Kristensson, P. O. (2013). Memorability of predesigned and user-defined gesture sets. In: Proceedings of the ACM SIGCHI Conference on Human Factors in Computing Systems (CHI), 1099-1108.

[15] Nielsen, J. (1993). Usability Engineering. San Francisco, CA.

[16] Nielsen, J.; Molich, R. (1990). Heuristic Evaluation Of User Interfaces. In: Proceedings of The ACM SIGCHI Conference On Human Factors In Computing Systems (CHI), 249-256.

[17] Reis, H. M.; Borges; S.; Isotani, S. (2013). Interação em interfaces de softwares de geometria interativa: Um mapeamento sistemático. In: Simpósio Brasileiro de Informática na Educação (SBIE), páginas 1-10.

[18] Reis, H. M.; Borges, S. S.; Durelli, V. H. S.; Moro, L. F. S.; Et Al. (2012). Towards Reducing Cognitive Load and Enhancing Usability through a Reduced Graphical User Interface for a Dynamic Geometry System: An Experimental Study. In: IEEE International Symposium on Multimedia, 445-450.

[19] Roanes-Lozano, E.; Roanes-Macías, E.; VillarMena, M. (2003). A Bridge Between Dynamic Geometry And Computer Algebra. Mathematical And Computer Modelling, 37(9-10), 1005-1028.

[20] Schimpf, F.; Spannagel, C. (2011). Reducing The Graphical User Interface Of A Dynamic Geometry System. ZDM, 43(3), 389-397.

[21] Shimomura, Y.; Hvannberg, E. T.; Hafsteinsson, H. (2013). Haptic Cues As A Utility To Perceive And Recognize Geometry. Universal Access In The Information Society, 12(2), 125-142.

[22] Trotta, F.; Imenes, L. M. P.; Jakubovic, J. (1979). Matemática Aplicada - Segundo Grau. 172 páginas.

[23] Vitale, J. M.; Swart, M. I.; Black, J. B. (2014). Integrating Intuitive And Novel Grounded Concepts In A Dynamic Geometry Learning Environment. Computers and Education, 72(March),231-248.

[24] Reis, H. M. ; Borges, S. S. ; Isotani, S. (2014). Análise de Usabilidade de Sistemas de Geometria Interativa para Tablets. RENOTE. Revista Novas Tecnologias na Educação, 12(1), 1-10.

[25] Blagojevic, R., Chen, X., Tan, R., Sheehan, R., \& Plimmer, B. (2012). Using tangible drawing tools on a capacitive multi-touch display. Proceedings of the Annual BCS Interaction Specialist Group Conference on People and Computers, $315-320$

[26] Blanke, D.; Schneider, G. (2011). TOM - A Multi-touch System for Learning Math. International Conference on Computer Supported Education, 199-206

[27] Jackiw, N. (1995). The Geometer's Sketchpad v3.0. Berkeley: Key Curriculum Press. 
[28] Ulrich Kortenkamp, (1999) Foundations of Dynamic Geometry, Ph.D. thesis, ETH Zurich, 11, http://kortenkamps.net/papers/diss.pdf.

[29] Brandão, L. O. (2002). Algoritmos e fractais com programas de geometria dinâmica. Revista do Professor de Matemática 49(2), 27-34.

[30] Guimarães, L. C.; R. Barbastefano; E. Belfort. (2002) Tools for synchronous distance teaching in geometry. In Proceedings of the International Conference on the Teaching of Mathematics, http://www.math.uoc.gr/ ictm2/Proceedings/pap 385.pdf.

[31] Kortenkamp, U.; and Richter-Gebert, J. (2004) Using automatic theorem proving to improve the usability of geometry software. Procedings of the Mathematical User-Interfaces Workshop. Available http://kortenkamps.net/papers/2004/ATP-UIarticle.pdf

[32] Santos, E.T.; Lourenzoni, L.; Oliveira, A. L. L. (2005) RISKO: desenhando com instrumentos virtuais. Simpósio Nacional de Geometria Descritiva e Desenho Técnico/V International Conference on Graphics Engineering for Arts and Design. Available at: http:/www.gente.eti.br/lematec/CDS/GRAPHIC A05/artigos/santoslourenzonideoliveira.pdf

[33] Oliveira, A. L. L. (2005). Avaliação comparativa de diferentes modelos de interfaces gráficas empregadas no ensino de geometria, segundo os conceitos de usabilidade. Dissertação de Mestrado, Universidade de São Paulo. Available at: http://www.teses.usp.br/teses/disponiveis/3/3146 /tde-10092007-091940/

[34] Isotani, S.; Brandão, L. O. (2008). An algorithm for automatic checking of exercises in a dynamic geometry system: iGeom. Computers and Education 51(3), 1283-1303.
[35] Kortenkamp, U.; Dohrmann, C. (2010). User interface design for dynamic geometry software. Acta Didactica Napocensia, 3(2). Available at http://dppd.ubbcluj.ro/adn/article_3_2_6.pdf

[36] Hinrichs, U.; Carpendale, S. (2011). Gestures in the wild: studying multi-touch gesture sequences on interactive tabletop exhibits. In Proceedings of the ACM SIGCHI Conference on Human Factors in Computing Systems, 3023-3032.

[37] Schimpf, F.; Spannagel, C. (2011) Reducing the graphical user interface of a dynamic geometry system. ZDM, 43(3), 389-397.

[38] Nielsen, J., and Landauer, T. K. (1993) A mathematical model of the finding of usability problems. Proceedings of ACM INTERCHI Conference, 206-213.

[39] Reis, H. M. ; Isotani, S. (2015). Concepção de uma Família de Gestos para Construção de Objetos Geométricos e sua Utilização em um Sistema de Geometria Interativa para Dispositivos Móveis: GeoTouch. In: Congresso Brasileiro de Informática na Educação, Maceió. Anais do Concurso de Teses Dissertações e Trabalhos de Conclusão de Curso (CTD-IE), 2015. p. 1-10.

[40] Isotani, S; Pedro, L. Z.; Reis, H. M; Borges, S. S.; Lopes, A. M. Z.; Souza, J. P. T.; Brandão, A. A. F.; Brandão, L. O. (2014) Interactive Geometry Goes Mobile with GeoTouch. Proceedings of the IEEE International Conference on Advanced Learning Technologies (ICALT), 181-185. 


\section{Apêndice A: Dicionário de gestos}

Ponto
Toque Breve
Toque brevemente com
odedo na tela

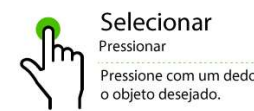

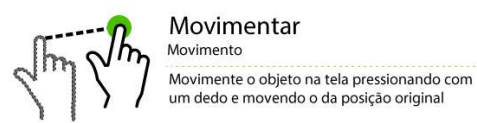

OTh ou

Refazer

年位a um objeto arrastando

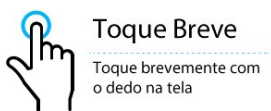

$S$ Arrasto

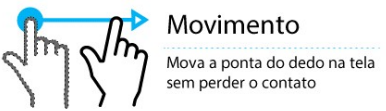

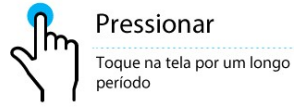

$\left\{\begin{array}{l}\text { Pressionar }+ \text { Toque Breve } \\ \begin{array}{l}\text { Pressione a tela com um dedo e depois } \\ \text { toque rapidamente a tela com o segundo dedo }\end{array}\end{array}\right.$

ou

ou 


\section{Apêndice B: Fluxograma}

Parte dos Fluxogramas para Construção de Objetos Geométricos Utilizando Gestos
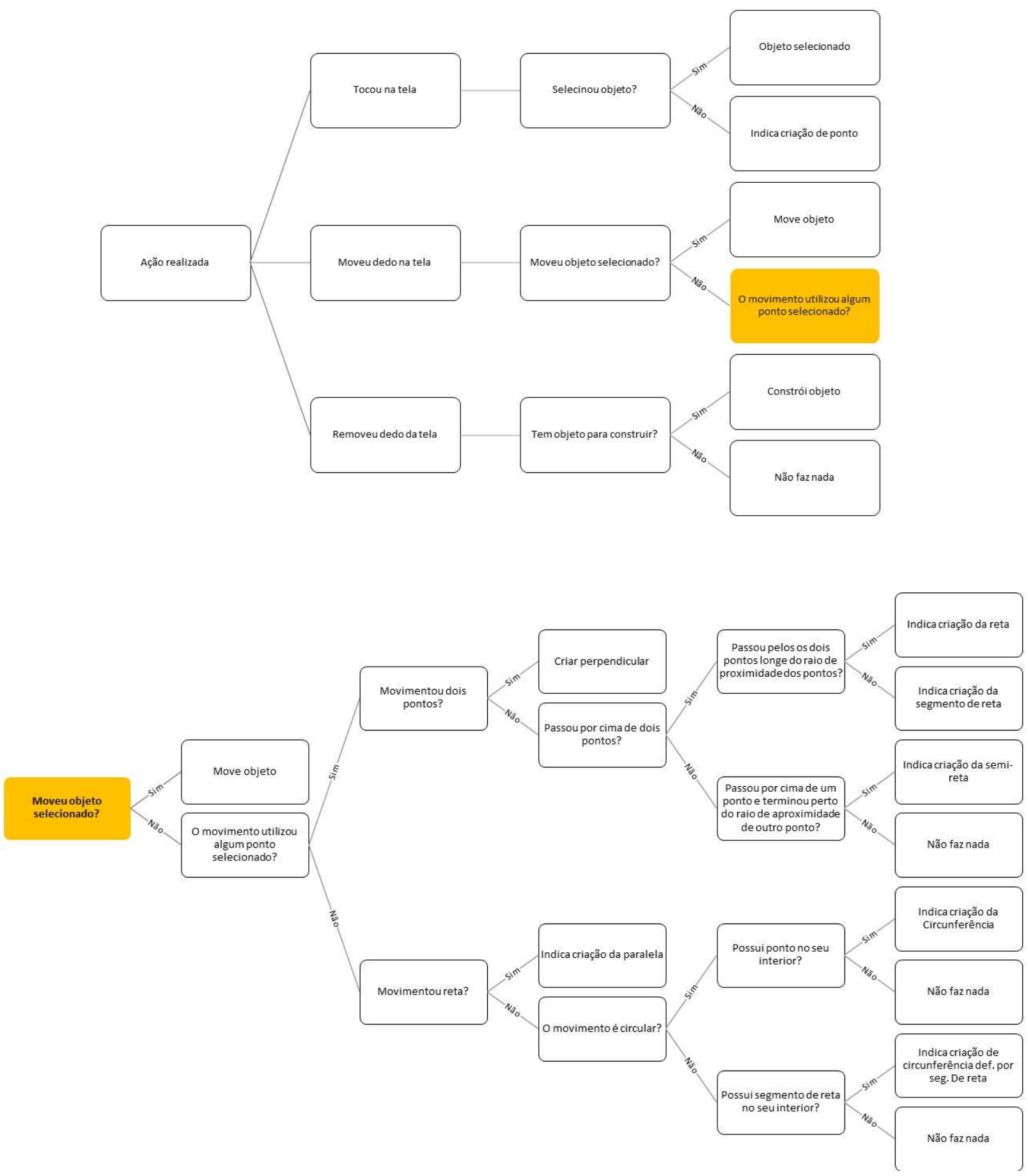


\section{Apêndice C: Entrevistas com professores de matemática}

1. Qual o seu nome, sua formação (incluindo onde estudou o doutorado e em qual área de pesquisa) e sua área/linha de pesquisa atual?

2. Quais são as dificuldades encontradas ao lecionar a geometria no modelo tradicional?

3. Quais os materiais utilizados em suas aulas de geometria?

4. Por que você optou pela utilização desses materiais?

5. Em quais cenários esses materiais são utilizados?

6. De qual maneira esses matérias melhoram no ensino?

7. Há algum problema encontrado nesses materiais?

8. Você usa ou ensina alguma tecnologia educacional (i.e. software de Geometria Interativa) para o ensino da geometria? Quais?

9. Em quais contextos (cenários) você os utiliza? Quais são as atividades?

10. Se não, qual contexto ou atividades utilizaria?

11. Você tem algum exemplo já pronto das atividades?

12. Por que você começou a usar o software de Geometria Interativa nas suas aulas?

13. A utilização deste software está funcionando nas aulas?

14. O que a utilização deste melhorou as aulas até agora?

15. Apresenta algum problema?

16. Há algum problema em termos de usabilidade?

17. O que poderia melhorar?

18. Se você criasse um sistema, qual(ais) funcionalidade(s) gostaria que possuísse? (O que mais faz falta ou o que seria interessante em trabalhar com os alunos).

19. Você acha que a interatividade é restrita na interface desses materiais e que prejudica no ensino da geometria?

20. Em termos de interatividade em relação aos materiais, o que poderia ser melhorado?

21. Você acha que uma interface natural ajudaria os alunos a aprimorarem o seu estudo da geometria? 